\title{
Diagnosis of smear-negative Pulmonary Tuberculosis in Kiffa hospital, Assaba, Mauritania
}

\author{
Boushab Mohamed Boushab ${ }^{*}$, Abdoulaye Mamadou Traore ${ }^{2}$,Mamoudou Savadogo ${ }^{3}$, Sidi Yeslem Ould-Bahiya ${ }^{4}$, \\ Fatima Zahra Fall-Malick ${ }^{5}$ \\ 'Médecine Interne et Maladies Infectieuses, Centre hospitalier de Kiffa, Assaba, Mauritanie \\ ${ }^{2}$ Service des Maladies Infectieuses, Hôpital du Point G, Bamako, Mali. \\ ${ }^{3}$ Service des Maladies Infectieuses, CHU Yalgado Ouédraogo, Ouagadougou, Burkina Faso \\ ${ }^{4}$ Service d'Imagerie Médicale, Centre National d'Oncologie, Nouakchott, Mauritanie. \\ ${ }^{5}$ Institut National d'Hépato-virologie, Faculté de Médecine de Nouakchott, Mauritanie
}

Article Info

\section{Article Notes}

Received: April 18, 2018

Accepted: May 16, 2018

\section{*Correspondence:}

Dr. Boushab Mohamed Boushab, Médecine Interne et Maladies Infectieuses, Centre hospitalier de Kiffa, Assaba, Mauritanie;

Telephone: (00222) 46749087 ;

Email: bboushab@gmail.com

(c) 2018 Boushab BM. This article is distributed under the terms of the Creative Commons Attribution 4.0 International License.

\section{Keywords}

Pulmonary TB

Diagnosis

Negative smear

Radiography

Mauritania

\section{ABSTRACT}

Background: In recent years, tuberculosis has been experiencing a renewal which appears to be linked to epidemiological, clinical and paraclinical factors. It is a real public health problem.

Summary: There is a prospective study at Kiffa regional hospital from January 1st to December 31st, 2017,

Objective: The goal was establish the real contribution of chest radiography to patient with respiratory infection diseases with a negative microscope smear.

Results: We recruited 53 cases of pulmonary tuberculosis (79\% of all TB cases). For Acid-Fast Bacilli (AFB) in sputum on direct examination and optical microscopy was negative in $52 \%$ of cases. The eight patients who accepted the HIV test, 6 were HIV positive. The sex ratio M/F was 1.7 and the average) age of patients was 45 years old (range 15-80 years)The most common clinical signs were fever, sputum, chronic cough and chest pain. The radiological aspects of the negative tuberculosis were caves (34\%), reticular or reticulonodular (26\%), lymph nodes pulmonary hilum opacities nodular (23\%), pleurisy (14\%) and miliary (3\%). In the lung lesion, parenchymal lung lesion were majority, it account for $64 \%$, more of the lesions were located in the right upper lobe( $49 \%)$.

Conclusion: This approach provided a TB diagnostic tool in patients with pulmonary tuberculosis negative bascilloscopie. In hospital practice, the combination of simple clinical, and radiological symptoms as an aid in the diagnosis of TB. Some Similar studies are needed to improve diagnosis in patients from outpatient suspected TB.

\section{Introduction}

Tuberculosis (TB) is a public health problem worldwide and recently abnormal more complex due to the persistence of aging populations and increasing emergence of drug resistant strains ${ }^{1}$. In resource-limited settings where sputum culture and nucleic acid amplification technology are not routinely test, diagnosis of pulmonary TB is based on physical examination, clinical signs, sputum smear microscopy ${ }^{2,3}$ and chest radiography ${ }^{2}$. The pulmonary TB with smear- negative represents $30-60 \%$ of all pulmonary TB cases, according to region ${ }^{4}$ and has little interest because it is less risk of contamination. In contrast, in clinical terms, it is a major concern, because of the importance of prognostic TB treatment ${ }^{5}$. The objective 
of the study is to investigate the contribution of chest radiography in the diagnosis of pulmonary tuberculosis smear-negative beyond the initial examination, patients received out patient at the reference of patients received out at the hospital of kiffa, in Mauritania) .

\section{Material and method}

\section{Frame and site of study}

Kiffa (104 506 inhabitants), the administrative capital of Assaba region (329 361 inhabitants), it's at $700 \mathrm{~km}$ from the capital Nouakchott. Assaba is located at the southeast of the country. Its Regional Hospital is the site of our study, that regional reference site offered a range of care and treatment in the specific areas of general surgery, pediatric surgery, orthopedics, infectious diseases, gastroenterology, cardiology, urology, hemodialysis, gynecoobstetrics and ophthalmology ${ }^{6}$.

\section{Patients and methods}

There is a prospective study of patients cared for pulmonary tuberculosis bacteriological confirmed at Kiffa regional hospital on the period 1 January 1st to December 31st, 2017 were included in the study, patients of higher age to 15 years and presenting a negative pulmonary TB bacilloscopy agreeing to participate in the study. Were excluded, the lower patients age 15 years presenting a lung infection other than tuberculosis. The collection of information was done with a listing of individual maintenance informed by an investigator after explaining the interest in the study and obtained the patient's consent. The diagnosis of pulmonary tuberculosis is usually conducted on an outpatient basis in all care services and hospitalization was proposed that in case of serious state (hemoptysis, Chest pain, Dyspnea...). Three sputum samples were applied to all patients with suspected pulmonary tuberculosis. The definition of pulmonary tuberculosis bacteriological confirmed was based on the instructions of Programme National de Lutte contre la Tuberculose (PNLT) with the presence of acidfast bacilli in at least two sample of sputum or a sample of sputum and X-rays pulmonary with evocative images of pulmonary tuberculosis. It's recorded smear-positive TB, without further investigation, any patient with at least two suptum bacteriological confirmed; was referred for chest radiography all smear-negative patients who did not improve after a trial of broad-spectrum antibiotics for 10 days and in which we had identified other causes of cough.

The decision to begin TB treatment is based on the interpretation of chest radiographsy. In this context of resource-limited settings when a clinician decides to put a patient under treatment (isoniazid, ethambutol, streptomycin, rifampicin, pyrazinamide), he refers it to Kiffa health center for notification the TB, taking charge and patient monitoring. The diagnosis of HIV infection was based on two rapid tests, the first sensitive and the second specific and discriminating.

\section{Results}

During the twelve-month of the study, 67 new cases of tuberculosis were identified. Pulmonary TB had $79 \%$ of all forms (53/67 case tuberculosis). The study focused only on the records of 53 patients who were able to do both chest radiography and at least direct sputum. For acid-fast bacilli (AFB) in sputum on direct examination by optical microscopy was positive in $27 \%$ of cases (18/67 patients) and negative in $52 \%$ of cases (35/67 patients). HIV serology was positive in six of the eight patients those agreed to do it. The sex-ratio Man / Woman were 1.7 and the average age was 45 years [15-80 years]. In all clinical signs of pulmonary TB have been dominated by the triad fever, chronic cough and sputum. The radiological anomalies encountered are caves, or reticular reticulonodular (Figure1), lymph nodes pulmonary hilum opacities nodular, pleurisy and miliary are respectively $34 \%, 26 \%, 23 \%, 14 \%$ and $3 \%$ (Table 1 ). The parenchymal lung lesion were dominated by localized damage (57\%), followed by numerous unilateral breaches (37\%) and bilateral involvement (6\%).

Table 1: Clinical relevance and radiographic abnormalities of 35 cases of pulmonary TB.

\begin{tabular}{|c|c|c|}
\hline $\begin{array}{l}\text { Clinical symptoms and radiographic } \\
\text { abnormalities }\end{array}$ & Frequency & Percent (\%) \\
\hline Clinical symptoms & 35 & 100 \\
\hline Fever & 28 & 80 \\
\hline Sputum & 21 & 60 \\
\hline Dyspnea & 20 & 57 \\
\hline Chronic cough & 11 & 31 \\
\hline Chest pain & 6 & 17 \\
\hline Hémoptysis & & \\
\hline Radiographic abnormalities & 12 & 34 \\
\hline Caves & 9 & 26 \\
\hline Nodular reticlo-opacity & 8 & 23 \\
\hline Mediastinal nodes / hilar & 5 & 14 \\
\hline Pleuresy & 1 & 3 \\
\hline Miliary & & \\
\hline
\end{tabular}

\section{Discussion}

TB is one of the most common infectious diseases in the world. It is the second leading cause of death from an infectious disease worldwide ${ }^{4}$, after the human immunodeficiency virus (HIV) ${ }^{1}$. In Mauritania, the limited diagnostic capacity for TB remains a challenge to improving case detection rates. Another limitation of our study is the limited sample which weakened the analysis of risk factors. However, the results of the study are important in the absence of similar information provided by previous studies and the challenges of achieving larger sample sizes in a difficult environment. Our study provides 


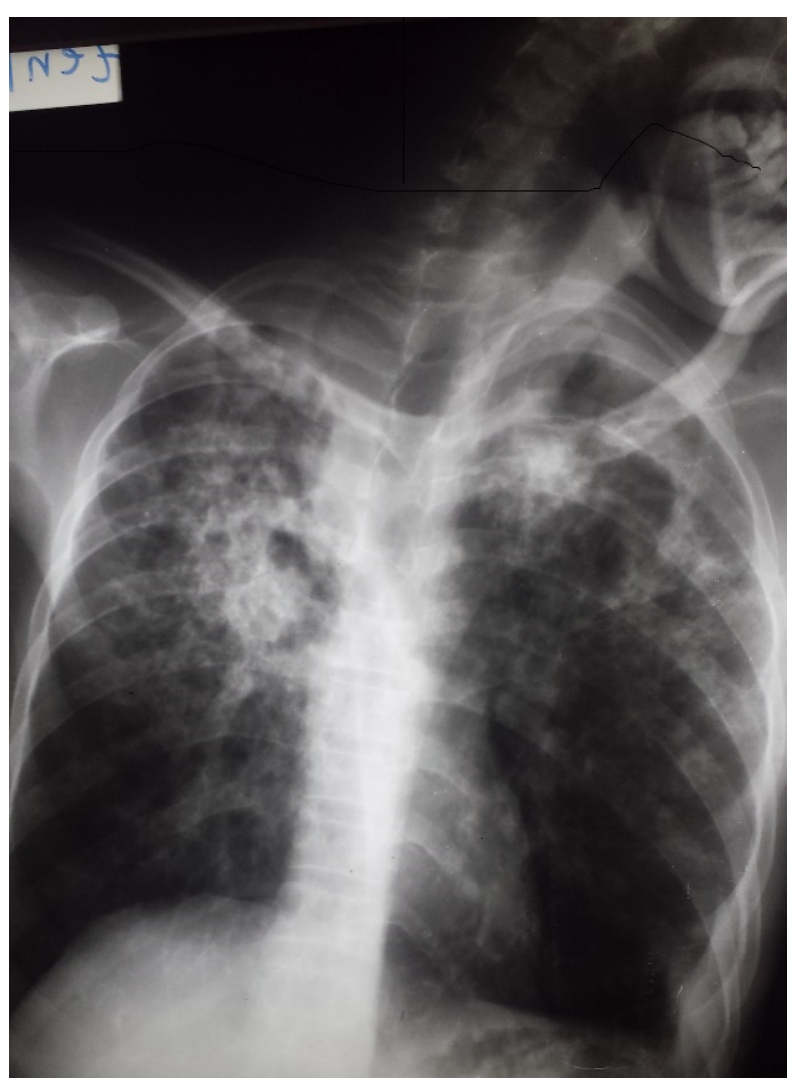

FIGURE 1 Infiltrates excavated from both lung fields with predominance at the apices

the first pulmonary tuberculosis prevalence data clinically diagnosed at the hospital of Kiffa and important information for strategic control of tuberculosis in the region. Pulmonary tuberculosis is $79 \%$ of TB cases. Among them, $52 \%$ were clinically diagnosed. We found a very high prevalence of clinically diagnosed pulmonary TB in our study. Previous investigations showed that the proportion cases of clinically diagnosed pulmonary TB ranged from $10 \%$ to $61 \%$ of all pulmonary TB cases $^{4,5}$. The HIV co-infection, considered a risk factor and worsening observed in six of the eight patients of cases who agreed to do it. This low number of serology is explained by the cost of the test and the refusal of some patients to be tested. For these reasons, the HIV serology was only performed in patients with symptoms suggestive. The potential differences in prevalence between smear negative, culture positive and clinically diagnosed, culture negative cases are possible due to several factors, including collection of unreliable sputum specimens, HIVcoinfection, duration of TB infection, the number of fields examined, the suspected cases population being examined, technical competence in sputum smearing and experiences to read smears ${ }^{5,6}$. This may be important as some studies have shown that HIV infection was associated with a higher probability of smear-negative TB disease ${ }^{7-10}$. Men were predominate $(63 \%)$. This data was reported by other authors in literature ${ }^{3,5,6}$. The analysis from some countries of the world, linked to the difficult working conditions in a context of widespread poverty, could be part of the explanation ${ }^{6}$. Clinically, the symptoms were very important at diagnosis significant. Radiologically, $57 \%$ of patients with pulmonary parenchymal lesions localized. Furthermore, $34 \%$ of lesions were carven. TB can present with symptoms and atypical radiologic findings that are indistinguishable from those of community-acquired pneumonia ${ }^{4}$, and the persistence of clinical and radiological abnormalities despite non specific antibiotic, the good conduct was considered a strong conjecture argument. The negative microscopy of sputum doesn't exclude the diagnosis of pulmonary tuberculosis, even if immediate changes on TB therapy were found to be favorable ${ }^{3}$. Among the case pulmonary TB clinically diagnosed, there are probably atypical mycobacterium infections. Chest radiography and sputum examination, despite its limitations, remains a critical step in diagnosing tuberculosis because they allow rapidly detect infectious cases.

\section{Conclusion}

Tuberculosisisa major publichealth problem worldwide, especially in developing countries. In Mauritania, as the countries with high TB prevalence and limited diagnostic facilities, the negative microscopy tuberculosis, should be considered in the persistence of suggestive clinical and radiological evidence of tuberculosis, the negativity of three sputum samples despite, not only specific antibiotic therapy is good conduct.

\section{Transparency Declaration}

The authors have no conflicts of interest to declare.

Author Contributions

All authors have read and approved the final manuscript.

Funding

Authors declare that they have no Funding.

\section{Acknowledgments}

We would like to acknowledge the tireless efforts from all the doctors for their good patients care at the Kiffa regional Referral hospitals. We thank for excellent technical assistance in laboratory diagnosis and radiography.

\section{References}

1. World Health Organization. Global tuberculosis report 2014. Geneva: WHO; publications. 2015;

2. Piatek AS, Van Cleeff M, Alexander H, et al. GeneXpert for TB diagnosis: planned and purposeful implementation. Glob Health Sci Pract. 2013; 1(1): 18-23.

3. Martínez-Romero A, Ortega-Sanchez JL, González-Yañez MG, et al. Comparison of acid-fast bacilli smear and chromatographic immunoassay in patients with active tuberculosis. Afr J Microbiol Res. 2012; 6(8): 1690-3. 
4. Campos LC, Rocha MVV, Willers DMC, et al. Characteristics of Patients with Smear-Negative Pulmonary Tuberculosis (TB) in a Region with High TB and HIV Prevalence. PLoS One. 2016 ; 25: 11(1).

5. Cavanaugh JS, Shah NS, Cain KP, et al. Survival among Patients with HIV Infection and Smear-Negative Pulmonary Tuberculosis - United States, 1993-2006. PLoS One. 2012; 7(10): e47855.

6. Boushab BM, Savadogo M, Sow MS. Prévalence de la tuberculose pulmonaire à bacilloscopie positive dans un centre hospitalier d'Aïoun (Hodh El Garbi). Rev Pneumol Clin. 2016; 72: 243-247

7. Affolabi D, Akpona R, Odoun M, et al. Smear-negative, culture-positive pulmonary tuberculosis among patients with chronic cough in Cotonou, Benin. Int J Tuberc Lung Dis. 2011; 15(1): 67-70.
8. Harries AD, Hargreaves NJ, Kwanjana JH, et al . Clinical diagnosis of smear-negative pulmonary tuberculosis: an audit of diagnostic practice in hospitals in Malawi. Int J Tuberc Lung Dis. 2001; 5(12): 114367

9. Hawken MP, Muhindi DW, Chakaya JM, et al. Under-diagnosis of smear-positive pulmonary tuberculosis in Nairobi, Kenya. Int J Tuberc Lung Dis. 2001 ; 5(4): 360-3.

10. Biadglegne F, Rodloff AC, Sack U. A First Insight into High Prevalence of Undiagnosed Smear-Negative Pulmonary Tuberculosis in Northern Ethiopian Prisons: Implications for Greater Investment and Quality Control.PLoS One. 2014 9; 9(9): e106869. 